\title{
Effect of miR-146a-5p on tumor growth in NSCLC using chick chorioallantoic membrane assay and bioinformatics investigation
}

\author{
WEN-TING HUANG ${ }^{1 *}$, WEI-LUAN CEN ${ }^{1 *}$, RONG-QUAN HE ${ }^{2}$, YOU XIE $^{1}$, YU ZHANG $^{1}$, \\ PING LI ${ }^{1}$, TING-QING GAN ${ }^{2},{\text { GANG } \mathrm{CHEN}^{1} \text { and XIAO-HUA HU }}^{2}$ \\ Departments of ${ }^{1}$ Pathology and ${ }^{2}$ Medical Oncology, The First Affiliated Hospital of \\ Guangxi Medical University, Nanning, Guangxi Zhuang Autonomous Region 530021, P.R. China
}

Received March 19, 2017; Accepted September 22, 2017

DOI: $10.3892 / \mathrm{mmr} .2017 .7713$

\begin{abstract}
Our previous study demonstrated that the expression of miR-146a-5p was downregulated in non-small cell lung cancer (NSCLC) tissue, which affected the progression and prognosis of patients with NSCLC. Thus, the present study was conducted to investigate the functional mechanism of miR-146a-5p in tumorigenesis and angiogenesis in NSCLC. Following the construction of a H460 NSCLC cell line in which miR-146a-5p was overexpressed via lentivirus transduction, the NSCLC chick embryo chorioallantoic membrane (CAM) model was established by transplanting miR-146a-5p-overexpressing NSCLC cells into the CAM. Then, the size of the neoplasms within the CAM was measured, the vessel ratio was calculated, and the cellular morphology, metastasis and inflammation of tumor cell was observed using hematoxylin and eosin staining. The target genes of miR-146a-5p were predicted by 12 online software programs; these genes were then subjected to Gene Ontology enrichment analysis and Kyoto Encyclopedia of Genes and Genomes pathway annotations using the Database for Annotation, Visualization and Integrated Discovery 6.7 as well as constructed into a protein interaction network using protein-protein interaction from Search Tool for the Retrieval of Interacting Genes/Proteins. The xenograft tumor size and angiogenesis conditions of the miR-146a-5p-overexpressing
\end{abstract}

Correspondence to: Professor Gang Chen, Department of Pathology, The First Affiliated Hospital of Guangxi Medical University, 6 Shuangyong Road, Nanning, Guangxi Zhuang Autonomous Region 530021, P.R. China

E-mail: chen_gang_triones@163.com

Professor Xiao-Hua Hu, Department of Medical Oncology, The First Affiliated Hospital of Guangxi Medical University, 6 Shuangyong Road, Nanning, Guangxi Zhuang Autonomous Region 530021, P.R. China

E-mail: gxmuhxh@163.com

*Contributed equally

Key words: NSCLC, miR-146a-5p, chicken embryo chorioallantoic membrane, tumorigenesis, gene prediction group (volume $6.340 \pm 0.066 \mathrm{~mm}^{3}$, vessel ratio $9.326 \pm 0.083$ ) was obviously restricted $(\mathrm{P}<0.001)$ when compared with the low expression group (volume $30.13 \pm 0.06 \mathrm{~mm}^{3}$, vessel ratio $16.94 \pm 0.11$ ). In addition, marked necrosis along with inflammatory cell infiltration was observed with the HE-stained slices from the miR-146a-5p low expression group. Regarding the results of the target gene prediction, cancer and toll-like receptor signaling were the two most significant pathways represented among the target genes, while JUN, EGFR and RAC1 were the most relevant proteins among the selected potential targets of miR-146a-5p. In a CAM xenograft tumor model, overexpression of miR-146a-5p inhibited the tumorigenesis and angiogenesis of an NSCLC cell line. miR-146a-5p may act as a tumor suppressor gene in NSCLC and have moderate prognostic value in lung cancer.

\section{Introduction}

Lung cancer has always been the most dominant cancer subtype. According to the latest statistics, the incidence and mortality of lung cancer have consistently occupied the top spot either in the country or worldwide and far outpaces other types of malignant tumors (1). Because there is no pertinent early diagnosis or optimal tissue-based molecular diagnostic procedure, lung cancer is usually in the advanced stages when diagnosed (2). Additionally, non-small cell lung cancer (NSCLC) accounts for $80 \%$ of the total number of lung cancer cases, including adenocarcinoma (ADC), squamous cell carcinoma (SCC) and large cell carcinoma (LCC) (3). Despite great efforts in elucidating the occurrence, development and prognosis of NSCLC in recent years, researchers still have not illuminated the potential tumorigenesis mechanism of NSCLC to date. As a consequence, studies are required to further develop a novel molecular-target diagnosis and therapy targeting NSCLC.

MicroRNAs (miRNAs), which are 20-25 nt in length, are a type of endogenous non-coding small RNA that regulates gene expression at either the messenger RNA (mRNA) or protein level during the post-transcription period $(4,5)$. miRNA can either inhibit the translation of mRNA or directly induce mRNA degradation by binding to the $3^{\prime}$ untranslated regions (3'UTRs) of mRNA (6); these processes can promote or restrain the proliferation, transformation, differentiation, 
apoptosis and necrosis of cells (6-8). Evidence has shown that abnormal miRNA expression is relative to the tumorigenesis process of several types of cancer (9), including NSCLC (10). miRNA has become the valuable biomarkers in a variety of diseases (11), especially cancer. miRNA can be used not only for NSCLC subtype analysis but also for monitoring the prognosis and recurrence of early stage NSCLC by identifying a specific sequence (12-16).

Previous studies had contrary conclusions that miR-146a acted to promote tumor growth in papillary thyroid carcinoma $(17,18)$ but also exerted tumor suppressor activities in malignancies located in the following organs: Breast (19), prostate (20-22), pancreas (23) and stomach (24-28). Regarding NSCLC, Wang et al reported that miR-146a had higher expression levels in NSCLC cells when compared with normal lung cells (29). Meanwhile, our previous study (30) concluded that miR-146a had low expression in NSCLC, but miR-146a mimic could inhibit cell proliferation and metastasis as well as induce apoptosis through the EGFR signaling pathway, which is in accordance with another published study (31). Therefore, we intended to detect the clinical significance and function mechanism of miR-146a-5p (abbreviated as miR-146a) in NSCLC.

In vivo animal experiment models have become an important means of NSCLC studies. To date, the chick embryo chorioallantoic membrane (CAM) model has become an efficient experimental animal model for studying tumors because it is cheap, convenient, fast, simple and sensitive. Because of its natural immunodeficiency and abundant formation of new blood vessels and an arteriovenous network, the CAM model is suitable for researching the mechanisms of angiogenesis, invasion and metastasis (32-39).

In this study, we intended to investigate the effect and molecular mechanism by which miR-146a-5p affects NSCLC using a constructed miR-146a-5p-expressing H460 NSCLC cell line and transplanting the transduced cells into the CAM of chick embryos. By establishing a CAM xenograft tumor model, we simulated the tumorigenesis process of NSCLC and observed the resulting angiogenesis. In addition, the local invasive and necrosis conditions of tumor were measured using hematoxylin and eosin (H\&E) staining. In addition, the prediction of target genes in silico also implicated the possible functional location and pathways of miR-146a-5p.

\section{Materials and methods}

Ethics statement. This research project was conducted with the permission of the Research Ethics Committee of the First Affiliated Hospital of Guangxi Medical University (Nanning, China).

Cell cultivation and selection. NSCLC cell lines provided by Yangjie Experiment Center of Guangxi Medical University consisted of two different histological types: The human lung large cell cancer $\mathrm{H} 460$, and the lung adenocarcinoma cancer cell lines A549, PC9 and H1299. The NSCLC cell lines were cultured in either RPMI-1640 medium (H460, A549 and H1299) or Dulbecco's modified Eagle's medium (DMEM) PC9 at $37^{\circ} \mathrm{C}$ in a humidified environment containing $5 \% \mathrm{CO}_{2}$. Detection of the miR-146a-5p expression levels in these lung cancer cell lines was performed to identify the cell line with the lowest expression levels of miR-146a-5p; this cell line (H460) was set aside for lentivirus transduction.

$R N A$ extraction and $q R T-P C R$. Operative steps for determining miR-146a-5p expression level were as follows. Total RNA was extracted using a total RNA extraction kit (no. 9767; Takara Biotechnology Co., Ltd., Dailan, China) according to the manufacturer's instructions. After determining the concentration and purity of the RNA by measuring the absorbance at 260 and $280 \mathrm{~nm}$, cDNA was generated by applying Takara Mir- $\mathrm{X}^{\mathrm{TM}}$ miRNA First-Strand Synthesis. The reactions were prepared using the SYBR ${ }^{\circledR}$ qRT-PCR User Manual. Primers were designed by using Online Prime 3.0, and then Invitrogen synthesized the primers.

Lentivirus transduction and interference efficiency verification. hsa-miR-146a lentivirus (LV-hsa-miR-146a) and a negative control lentivirus (LV-no load, LVCON238) were both purchased from the Shanghai Jikai Gene Chemical Co., Ltd. (Shanghai, China) and stored at $-80^{\circ} \mathrm{C}$. The target sequence of miR-146a-5p (miRBase accession, MI0000477) was TGAGAACTGAATTCCATGGGTT, and the vector was GV369, which contained the component order Ubi-MCS-SV40-EGFP-IRES-puromycin. The sequence of the negative control was TTCTCCGAACGTGTCACGT. During the transduction process, the selected cell line H460 was divided into three groups: Blank control group (untreated non-transduced H460 lung cancer cell line), the experimental group (LV-hsa-miR-146a group) and the negative control group (LV-no load, LVCON238). Cell morphology and fluorescence intensity were monitored under the fluorescence microscope at 48 and $72 \mathrm{~h}$ after transduction, respectively. Finally, total RNA was extracted from the cell lines, and qRT-PCR was applied to quantify the relative miR-146a-5p expression.

Chick embryo preparation. Fertilized chick embryos were purchased from Nanning Liangfeng Agriculture and Farming Company Limited. After it was sterilized in $75 \%$ alcohol, the chick embryo was placed in an incubation box (which was also sterilized by $75 \%$ alcohol) at $37.6-38^{\circ} \mathrm{C}$ with $70-80 \%$ humidity. The chick embryos were incubated until they were 8-day-old for experimental use.

CAM tumor xenograft assay. i) When the chick embryos were 7 days old, the contours of the embryoid body, gas chamber and large blood vessels attached to the chick chorioallantoic membrane on the egg shells were sketched under the exposure of an egg tester on sterile platform. ii) When the chick embryos were 8 day-old, they were removed and sterilized with $75 \%$ alcohol. A 2-mm diameter hole was drilled into the gas chamber side of every egg, and the embryo egg membrane was pricked with a fine needle. Next, an approximately $2 \times 1 \mathrm{~cm}$ window was ground out on the egg shell near the embryoid body and large blood vessels to expose the white membrane. Afterwards, air was repeatedly suctioned through the previously drilled hole into the gas chamber to isolate the white membrane and chick chorioallantoic membrane. When the white membrane was removed to expose the vessels, a $5 \mathrm{~mm}$ silicon rubber ring was gently placed above the vessel rich 


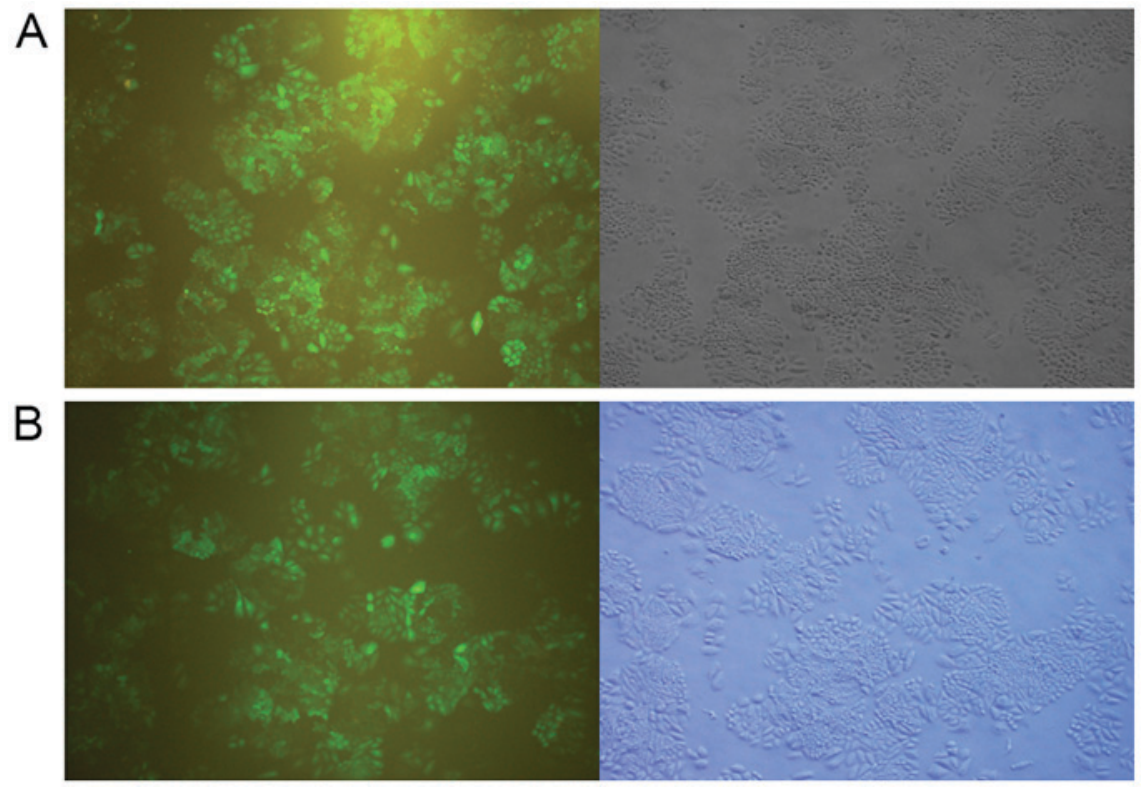

Figure 1. The transduction outcomes of both H460 cells groups treated with lentivirus. The cells were transduced with (A) LV-hsa-miR-146a or (B) negative control. All cells were observed under a light microscope (x200).

area on the CAM. Finally, the small hole in the gas chamber was sealed up with sterile transparent materials. The above procedure was conducted on a sterile platform. iii) A cell suspension solution from each group was gently added to the silicon rubber ring, and the window was sealed using sterile transparent materials. After incubating for $24 \mathrm{~h}$ in the incubation box, the silicon rubber ring was removed, and the model system was then placed back into the incubation box to incubate for $120 \mathrm{~h}$. Throughout this duration, the tumorigenesis condition was monitored and recorded every $24 \mathrm{~h}$. iv) After $120 \mathrm{~h}$, the chick embryo and sterile transparent materials were discarded, and the xenograft tumors were removed from the CAM. These tumors were photographed and measured to record their size. v) The vessel ratio and window's area were estimated by using Image-Pro Plus. vi) After embedding the tumors with paraffin, the samples were sliced and stained with HE-staining to observe the cellular morphology of tumor cell as well as the metastasis conditions.

Identifying potential target genes in silico and bioinformatics analysis. The predicted and validated microRNA gene targets were obtained from miRWalk2.0 (http://zmf.umm. uni-heidelberg.de/apps/zmf/mirwalk2/). The twelve prediction databases were miRWalk, MicroT4, miRanda, mirbridge, miRDB, miRMap, miRNAMap, PicTar2, PITA, RNA22, RNAhybrid and TargetScan, and the three validated databases were miRTarBase, TarBase and miRecords. For predicting the target genes, those ones which were repeated in four or more databases were selected for the next step. The overlapping hits of the selected predicted genes and all the validated genes were entered into a gene-enrichment pathway analysis. Function analysis of the potential target genes from the Kyoto Encyclopedia of Genes and Genomes (KEGG) pathway annotations and Gene Ontology (GO) terms were provided by Database for Annotation, Visualization and Integrated Discovery 6.7 (DAVID 6.7, https://david.ncifcrf.gov/) (40). The protein interaction analysis was showed by protein-protein interaction (PPI) Networks from Search Tool for the Retrieval of Interacting Genes/Proteins (STRING, http://www.string-db. $\operatorname{org} /)$.

Statistical analysis. Experimental data were analyzed using SPSS 20.0 (SPSS, Inc., Chicago, IL, USA) statistical software using methods such as the Independent Samples t-test, $\chi^{2}$ test and Spearman's correlation coefficient analysis, and $\alpha=0.05$ was considered as the test threshold. When the two-sided P-values was $<0.05$, the result was considered to indicate a statistically significant difference.

\section{Results}

The expression levels of miR-146a-5p in 4 cell lines. Total RNA extracted from the H460, PC9, 1299 and A549 cell lines were analyzed for concentration and purity. H460 cells presented the lowest expression level of miR-146a-5p than the other three cell lines; thus, H460 cells were selected to construct the CAM xenograft tumor model. The relative expression levels of miR-146a-5p in the 4 NSCLC cell lines were showed that H460 cells had the lowest miR-146a-5p expression among the cell lines tested.

Lentivirus transduction outcomes. H460 cells were transduced with the optimal ratios for either LV-hsa-miR-146a or LV-no load (Fig. 1). The miR-146a-5p expression levels in H460 cells transduced with LV-hsa-miR-146a and LV-no load group were detected by qRT-PCR. The results showed that the H460 cells were successfully transduced with lentivirus and that the miR-146a-5p in LV-hsa-miR-146a groups presented obviously overexpression than the negative LV-no load group.

CAM xenograft tumor model. The results of the xenograft tumor sizes from day 1 ( $24 \mathrm{~h}$ after inoculation, or day 8 of 


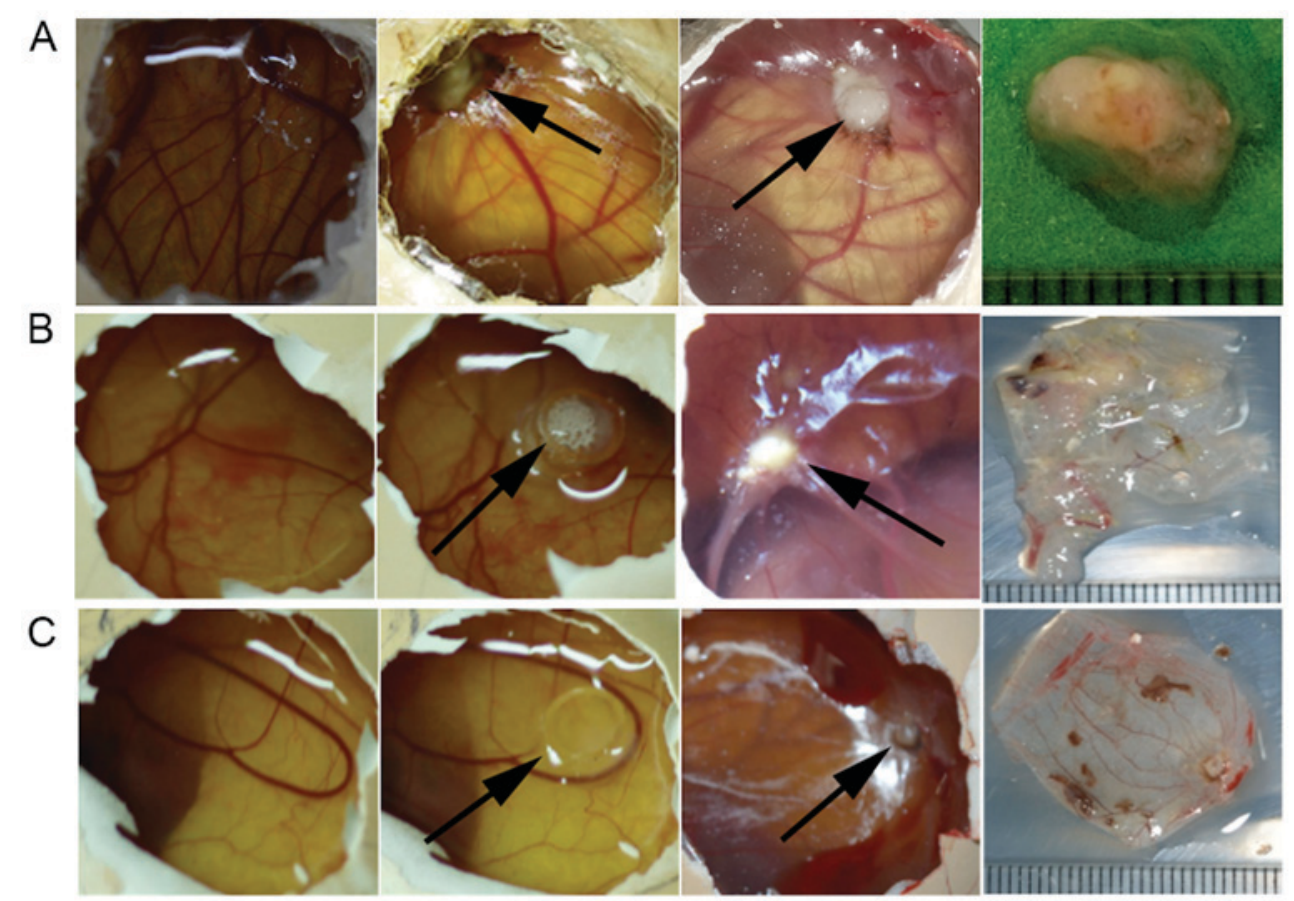

Figure 2. Tumor formation of (A) blank H460 lung cancer cells transduced with (B) either LV-no load or (C) LV-hsa-miR-146 in a chick chorioallantoic membrane.
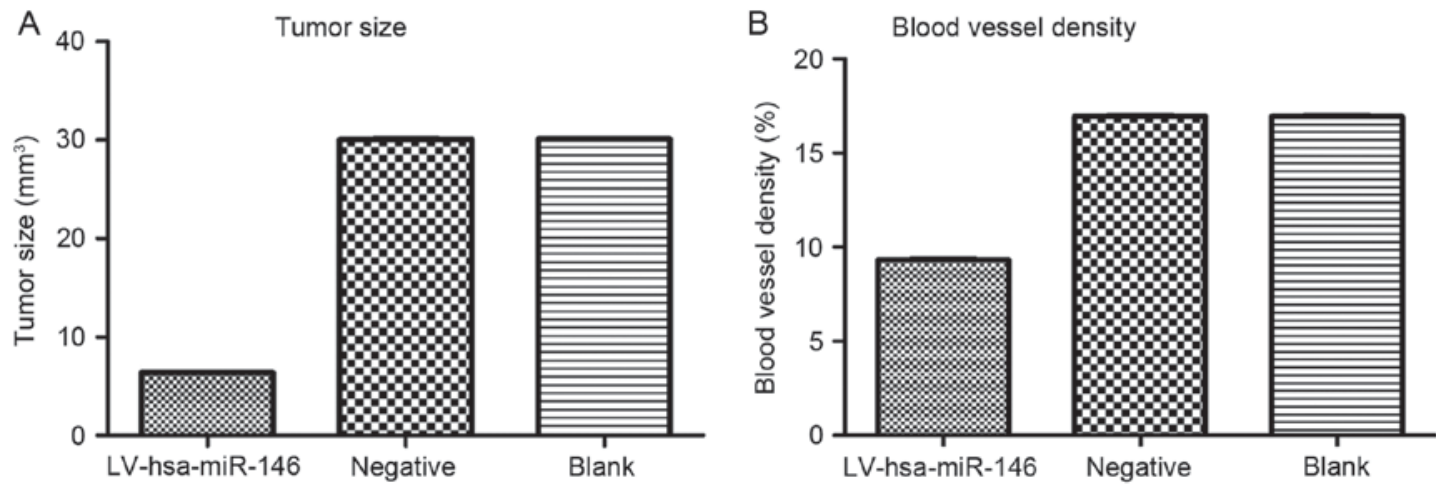

Figure 3. The chick chorioallantoic membrane (CAM) of tumor formation and angiogenesis. (A) Tumor formation of blank H460 cells and H460 cells transduced with either LV-no load (negative) or LV-hsa-miR-146 in CAM. (B) Overexpression of miR-146a-5p inhibited angiogenesis of tumors xenograft in CAM.

chick embryo growth) to day 5 (120 h after inoculation, or day 12 of chick embryo growth) were showed in Figs. 2 and 3. First, one-way analysis of variance (ANOVA) was performed, but the results were not statistically significant. Then, the independent sample t-test was performed by comparing the sizes with those of the blank control group. The size of the xenograft tumors in the experimental group (LV-hsa-miR-146a group, $\mathrm{V}=6.340 \pm 0.066 \mathrm{~mm}^{3}$ ) were obviously reduced compared with that in the blank control group (untreated H460 cell line, $\left.\mathrm{V}=30.13 \pm 0.06 \mathrm{~mm}^{3}\right)(\mathrm{t}=613.489, \mathrm{P}<0.001)$, and the tumor size of the negative control group $\left(\mathrm{V}=30.09 \pm 0.07 \mathrm{~mm}^{3}\right)$ exhibited no statistical significance when compared with the blank control group (untreated $\mathrm{H} 460$ cell line, $\mathrm{V}=30.13 \pm 0.06 \mathrm{~mm}^{3}$ ) $(\mathrm{t}=1.312, \mathrm{P}=0.260)$.

Angiogenesis of xenograft tumors. Based on the duration of the observed incubation period and final angiogenesis data
(Fig. 3), the independent samples t-test was performed by comparing the results of the blank control group with the non-significant result of the one-way ANOVA. The appearance of the xenograft tumors on day 1 was an attached tumor on the CAM with a few capillary vessels thriving and surrounding the uneven surface of the xenograft tumor; by day 5, the angiogenesis conditions of the experimental group (LV-hsa-miR-146a group, 9.326 \pm 0.083 ) were largely reduced compared with those of the blank control group (untreated H460 cells, 16.94 \pm 0.11 ) $(\mathrm{t}=121.207, \mathrm{P}<0.001)$, while growth situation of vessel showed no significant difference between the negative control group (LV-no load group, 16.97 \pm 0.07 ) and the blank control group (untreated H460 cells, 16.94 \pm 0.11$)(t=-0.612, \mathrm{P}=0.573)$. Since tumor growth relies on the generation of blood vessels in the chick CAM model, the overexpression of miR-146a-5p in the experimental group inhibited the angiogenesis of the xenograft tumors and thus restrained tumor growth. 

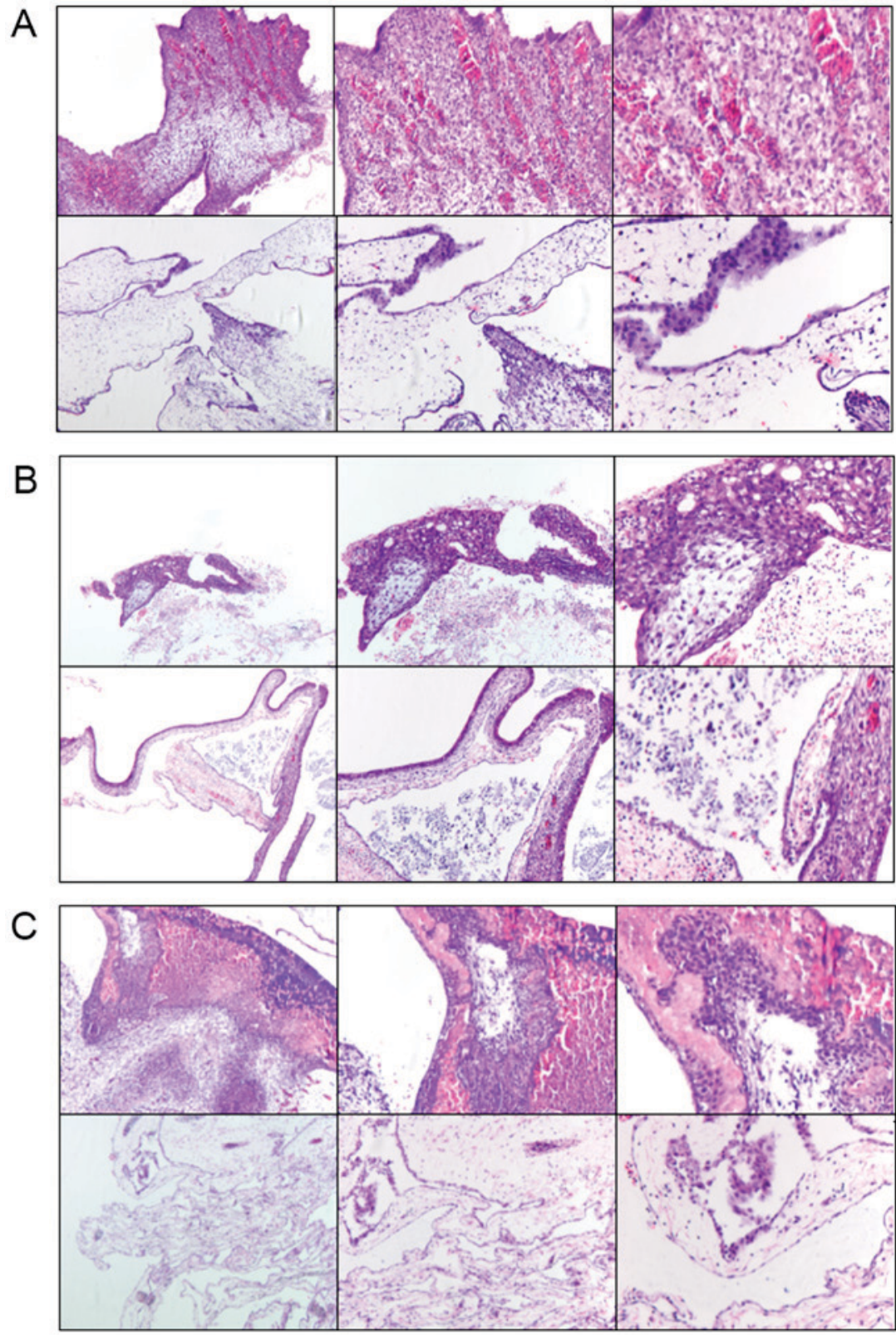

Figure 4. Detection of the influence of miR-146a-5p overexpression on tumor formation using the chick chorioallantoic membrane (CAM) model [hematoxylin and eosin staining (H\&E)]. (A) Blank H460 cells; (B) H460 cells transduced with negative control; and (C) H460 cells transduced with LV-hsa-miR-542 (from left to right in the order: 100, 200 and x400 magnification).

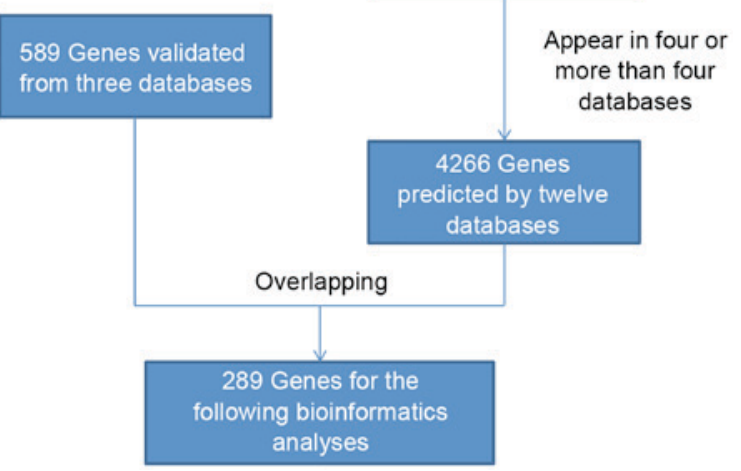

Figure 5. Flow diagram of the bioinformatic prediction of miR-146a-5p target genes.
$H \& E$ staining. Tumor tissue was removed, paraffin-embedded and subjected to HE-staining to observe the cell morphology (Fig. 4). Under a light microscope, all three groups could form tumors on the CAM and showed preservation of the primitive morphology of cancer, including obvious necrotic areas and inflammatory cell infiltration. However, the inflammatory condition of the experimental group (LV-hsa-miR-146a group) was relatively inconspicuous compared with that of the blank control group (untreated H460 cells).

Potential target genes of miR-146a-5p based on bioinformatics analysis. Twelve in silico interaction tools to predict miRNA targets were used in this study. The databases were miRWalk, MicroT4, miRanda, miRBridge, miRDB, miRMap, miRNAMap, PicTar2, PITA, RNA22, RNAhybrid and TargetScan. In total, 14,278 target mRNAs were listed as 
Table I. Potential target genes of miR-146a-5p.

\begin{tabular}{|c|c|c|c|c|c|c|c|}
\hline IER5L & EIF4G2 & SMAD4 & STC1 & CCDC6 & MTA2 & PHF20L1 & LCOR \\
\hline CCDC117 & THAP5 & ELAVL1 & IRAK1 & NFIX & PRKCE & TCF20 & BTG2 \\
\hline SYNJ1 & AP3S2 & FNBP4 & FBXO3 & BRWD1 & C12orf4 & SRPRB & TRAK2 \\
\hline KCTD15 & ATG9A & CDC73 & TMEM167A & ZNF367 & HIPK1 & ACTBL2 & PTGS2 \\
\hline RAC1 & TIMELESS & RHOBTB3 & BRK1 & ITCH & ATP5G2 & SLC26A2 & EGFR \\
\hline ERBB4 & NR6A1 & MYO6 & NF2 & ROBO1 & PPP1R11 & TSPYL1 & CASK \\
\hline NUMB & SERTAD2 & MED13 & WASF2 & CARD8 & MKRN2 & FBXL3 & C16orf72 \\
\hline CARD10 & FAM8A1 & TMEM214 & PLEKHG5 & EPB41L4A & NSD1 & DDHD1 & TSPAN14 \\
\hline VANGL1 & NACC1 & ARL8A & SESN3 & SAMD9L & TMEM136 & CD80 & CD40LG \\
\hline CDKN3 & EIF4EBP2 & $\mathrm{CFH}$ & MR1 & HSPA1A & IFIT3 & PMAIP1 & POU3F1 \\
\hline PSMD3 & RBL1 & SFRP1 & SLC2A3 & STAT1 & SF1 & LSM4 & METTL7A \\
\hline RUFY2 & STARD7 & RFX7 & LIMD2 & MOB1B & ZNF257 & EDARADD & WDR36 \\
\hline PTAR1 & CCDC83 & EFNA5 & FANCF & IRF5 & LBR & MID1 & TLL1 \\
\hline TRAF6 & BCL7B & HYOU1 & ARPP19 & ZHX1 & RAB18 & AVL9 & PDS5A \\
\hline TNRC6A & BABAM1 & TULP4 & GOPC & ATP13A3 & EDEM3 & TMPRSS5 & SLC38A1 \\
\hline STK40 & RHPN2 & TMEM67 & SESTD1 & EPSTI1 & NACC2 & HORMAD2 & OLFML2A \\
\hline SKA2 & TMPPE & BRCA1 & BRCA2 & CD86 & GART & GPM6B & SMAD2 \\
\hline MKLN1 & MVD & PPP2R4 & CCL5 & SDCBP & SLC1A5 & UMPS & CDS2 \\
\hline CD84 & AKAP8 & CCR9 & AAK1 & ZNF629 & ZNF117 & ZDHHC13 & GIMAP4 \\
\hline PBLD & C1orf21 & UTP15 & USP48 & ST6GAL2 & RAB2B & ZNF493 & ZNF260 \\
\hline TRIM22 & IGF2BP1 & ERRFI1 & MFSD6 & RAB20 & APMAP & GATAD2B & RAPH1 \\
\hline PARD6B & BACH1 & CALU & COPA & GNAI2 & HOXB8 & IREB2 & NF1 \\
\hline NFE2L1 & RORA & SRPK1 & RAD54L & SQSTM1 & CPNE3 & DEDD & ZBTB22 \\
\hline SLK & TXNIP & SERBP1 & RBM26 & PAPD5 & GRPEL1 & ISG20L2 & RASSF5 \\
\hline TMEM101 & ITPRIPL2 & UBN2 & ATXN1L & C16orf52 & AKT2 & LY75 & POLR2E \\
\hline TLR4 & TPD52 & ZNF264 & NUPL1 & BTN2A2 & COPS8 & SNRNP27 & CARHSP1 \\
\hline STMN3 & AEN & SIKE1 & ATOH8 & SYT12 & VWCE & PLIN2 & HLA-C \\
\hline LAMC2 & STIM1 & ULK1 & MSC & RAPGEF5 & MDFIC & PPHLN1 & CYBRD1 \\
\hline DGCR6L & SLFN11 & PRR15 & PA2G4 & KLF9 & CAPN2 & JUN & TNPO1 \\
\hline LNPEP & RPL11 & CCL8 & SOX4 & WASL & LATS1 & DDX21 & ZBTB33 \\
\hline G3BP1 & SUPT16H & MDN1 & STX12 & IFIT5 & CLIC4 & KLHL20 & $\mathrm{NDC1}$ \\
\hline KIAA1432 & SHCBP1 & KLHL15 & CHMP4B & MTPN & DYNLL2 & CPNE8 & IFIT1 \\
\hline LTB & FXR1 & KIAA0040 & ATP11B & PACS2 & UHRF1 & HM13 & MRPL10 \\
\hline ALG10B & ADD1 & CCNA2 & ELK4 & MYLK & SERPINB9 & NAPG & PAPOLA \\
\hline LMTK2 & CBX6 & SLC22A15 & TXLNG & KLHL42 & ACBD3 & DBF4B & KBTBD6 \\
\hline USP54 & CNOT6L & ZNF410 & P2RX5 & RAG1 & CXCL12 & RER1 & ETNK1 \\
\hline TET3 & & & & & & & \\
\hline
\end{tabular}

predicted genes. Eight genes were predicted by ten databases in silico (FZD3, KLF7, STRBP, ZBTB2, IQGAP3, UPP2, JAZF1 and IER5L), which provides strong evidence that these genes are targets of miR-146a-5p. To improve the credibility of these target genes, we obtained 4,266 intersecting elements that were simultaneously predicted by at least four databases. Then, these elements were overlapped with all 589 validated target genes of miR-146a-5p using the databases miRTarBase, TarBase and miRecords to finally obtain 289 target genes (Fig. 5; Table I).

The top 10 most significant KEGG pathway annotations were showed in Table II. The most significant KEGG pathway was involved in cancer. Moreover, the top $10 \mathrm{GO}$ terms of biological process (BP), cellular component (CC) and molecular function (MF) are listed in Table III, and the first terms of each analysis were cellular process, cell part and binding, respectively. The network of BP and CC are shown in Figs. 6 and 7. The most significant KEGG pathway (hsa05200, pathways in cancer) is shown in Fig. 8. The PPI network included 50 hub genes (Fig. 9). JUN, EGFR and RAC1 were the most relevant protein among the selected possible targets of miR-146a-5p.

\section{Discussion}

Lung cancer has been consistently regarded as the most aggressive carcinoma worldwide and accounts for a large percentage of cancer-related deaths (41). Although there is substantial amelioration in the application of chemical and molecular-targeted treatments, the prognosis of patients with 


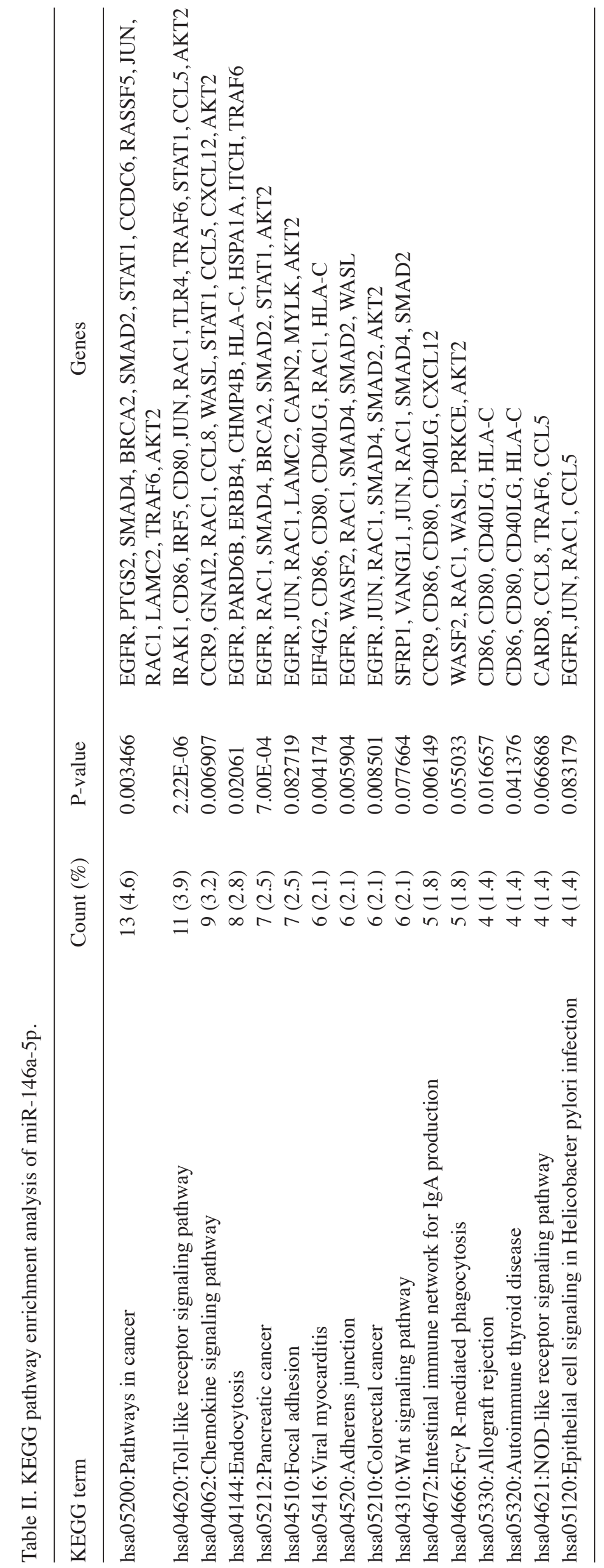




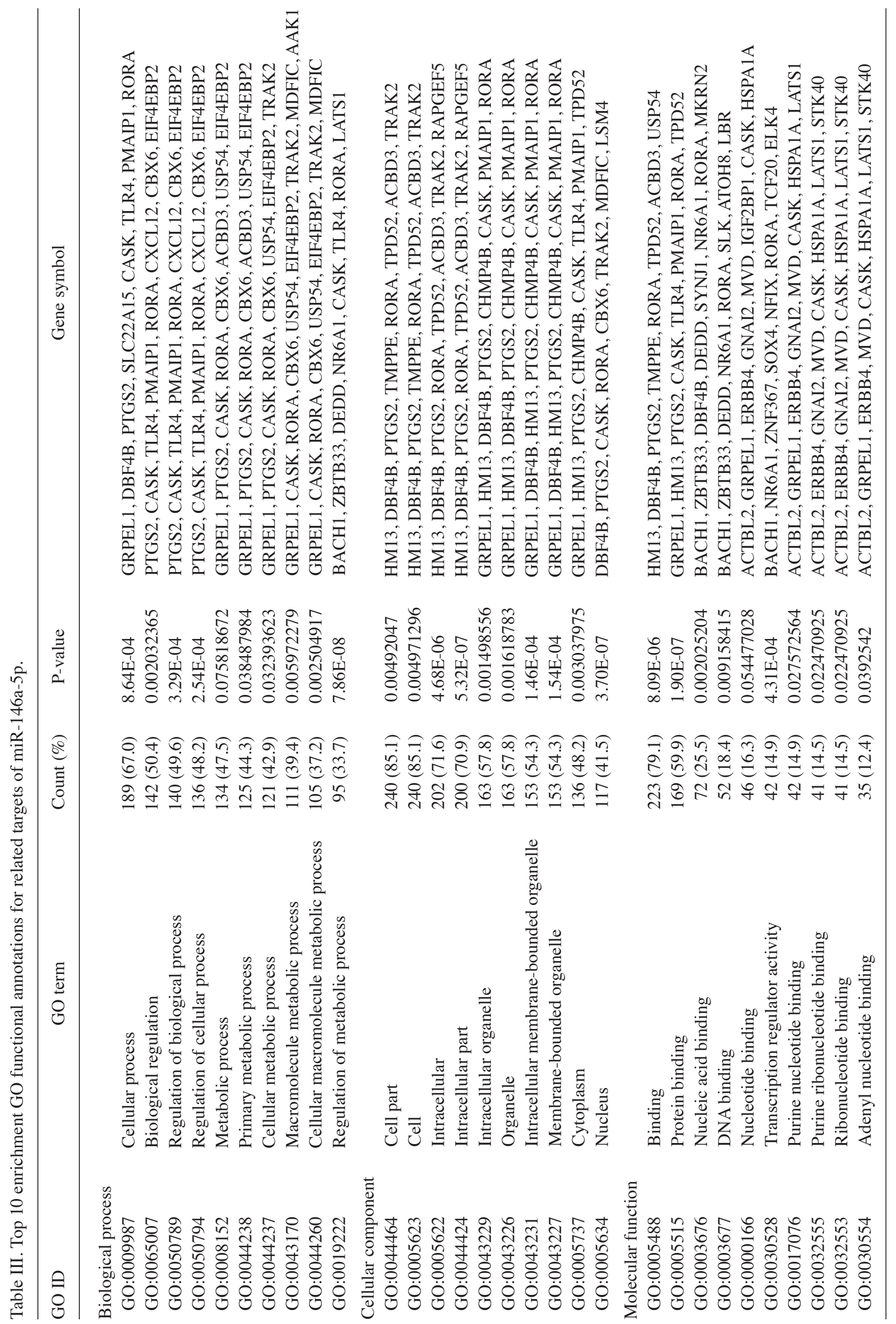




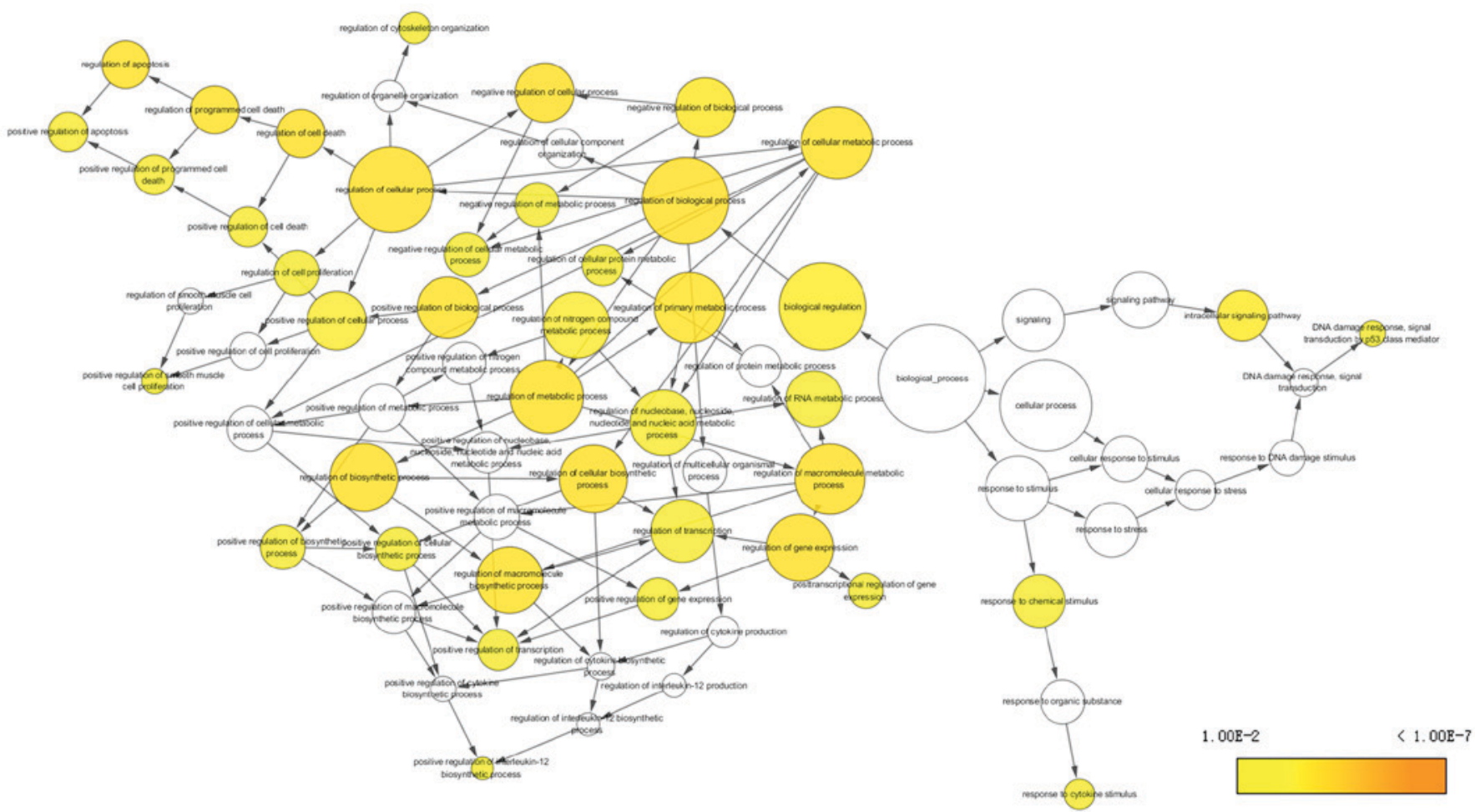

Figure 6. Network analysis with the prospective target genes of miR-146a-5p of BP. The intensity of the color indicates P-value size, node refers to pathways, and the node size is representative of the number of genes.

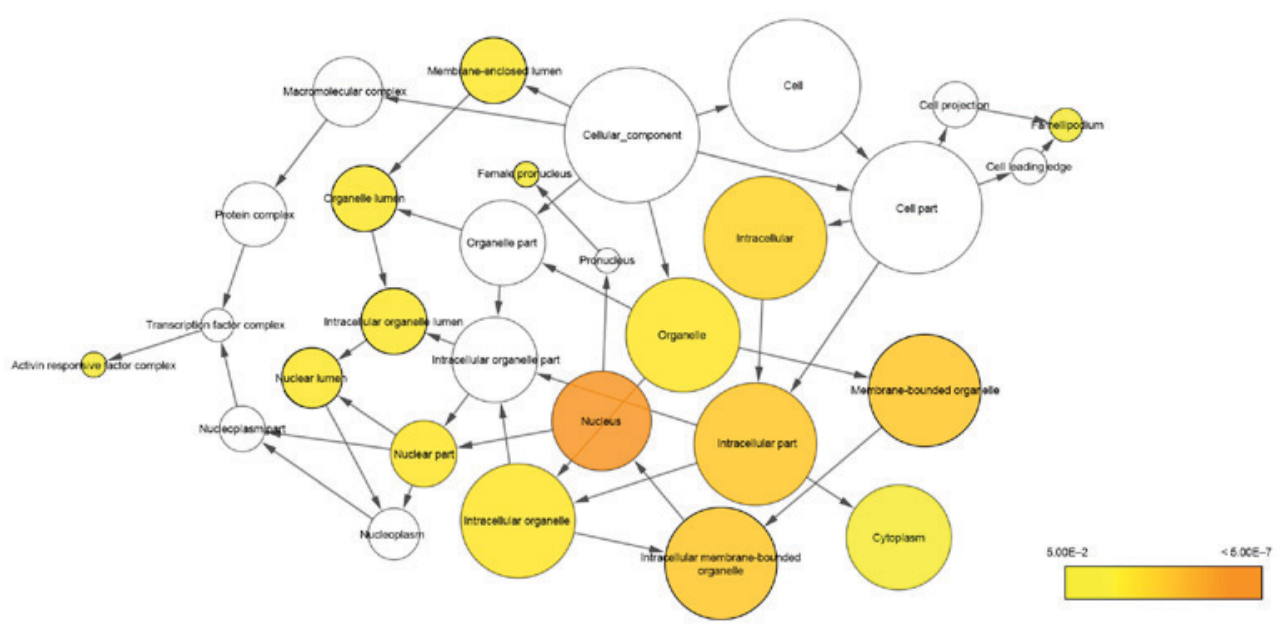

Figure 7. Network analysis with the prospective target genes of miR-146a-5p of CC. The intensity of the color indicates P-value size, node refers to pathways, and the node size is representative of the number of genes.

lung carcinoma is still embarrassing (42). Since the consensus behaviors of cancer cells-invasion and metastasis-are the major hurdles for the clinical treatment of NSCLC, more attention should be focused on the verification of small molecules that can suppress the invasion, metastasis and angiogenesis of NSCLC.

Recently, evidence demonstrated that miRNAs can be used as diagnostic and prognostic biomarkers of leukemia, lung cancer and colon cancer (43). miRNAs may also be new therapeutic agents for antitumor therapies in humans (44). One study found that miRNAs can regulate the signal transduction of the EGFR signaling pathway in a wide variety of tumor cells (45), including lung cancer (46). Identifying the molecules within the EGFR signaling pathway that are targeted by miRNAs may be a potential therapeutic approach for treating lung cancer. Previously, we found that low expression of miR-146a-5p in NSCLC cells inhibited cell proliferation and metastasis as well as induced apoptosis through the EGFR signaling pathway by using functional experiments (30); these results were concomitant with another study conducted by $\mathrm{Li}$ et al (31). In contrast to other studies on miR-146a-5p expression in NSCLC, this study verified the reliability of our previous experimental study in vivo to identify differences of biological growth properties and molecular variations among the CAM xenograft tumors comprising blank control, negative control or experimental cells. 


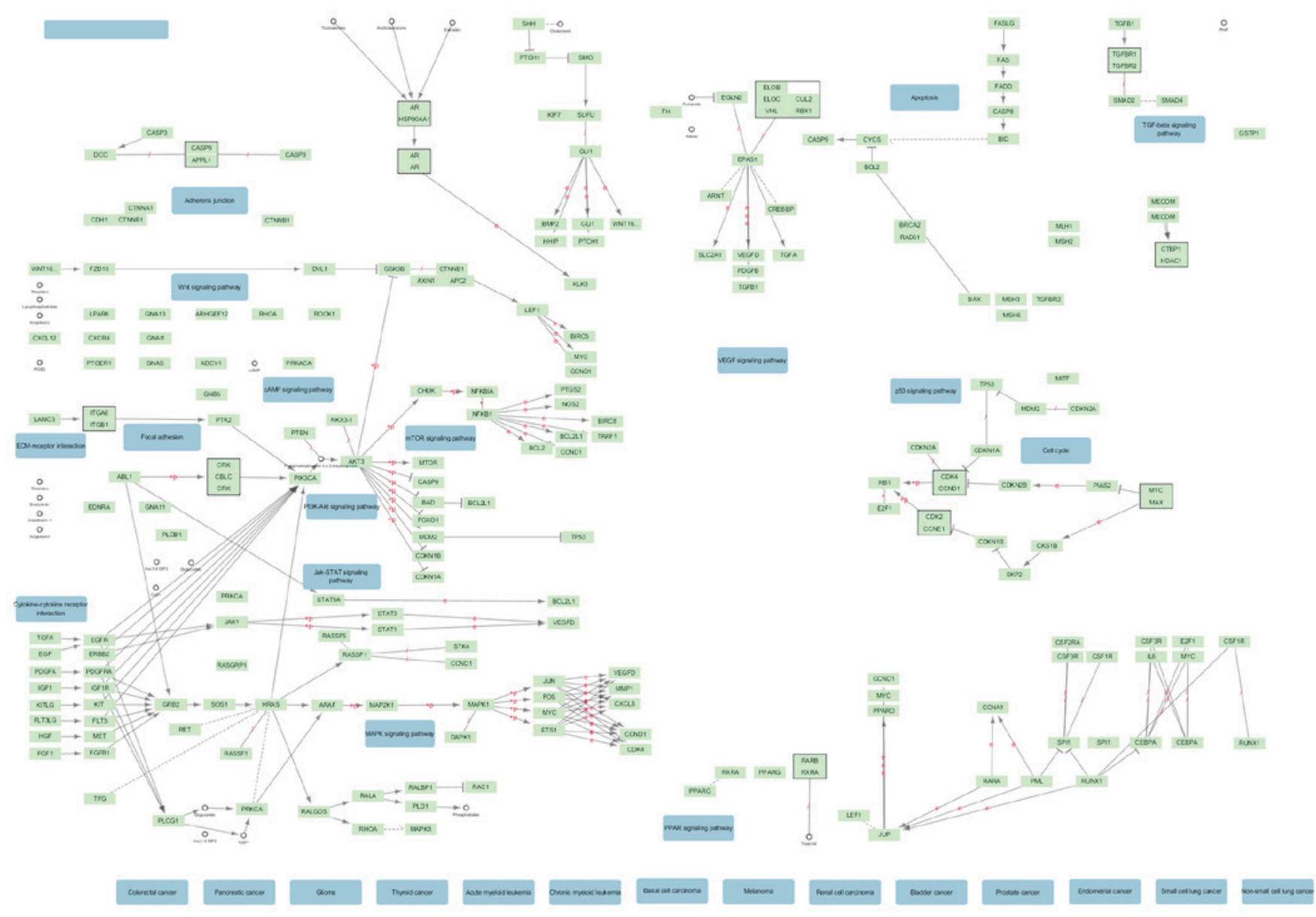

Figure 8. The top one Kyoto Encyclopedia of Genes and Genomes (KEGG) pathway enrichment analysis of miR-146a-5p, hsa05200: Pathways in cancer.

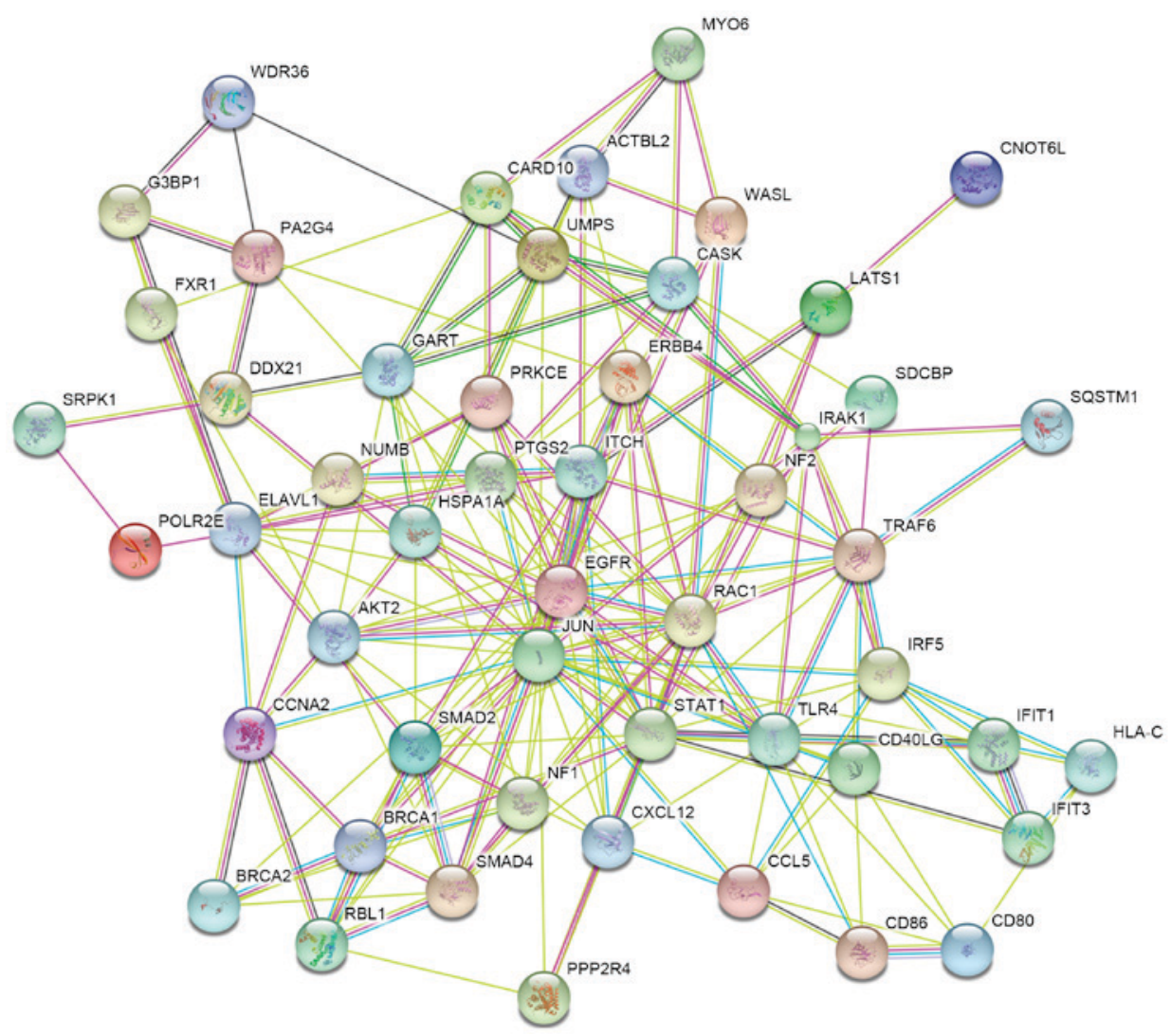

Figure 9. Hub gene protein-protein interaction (PPI) networks of the potential mRNAs targeted by miR-146a-5p. 
Our previous study identified EGFR as a downstream regulatory target of miR-146a at both the mRNA and protein level (30). The high expression of miR-146a inhibited the proliferation of NSCLC cells by downregulating the expression of EGFR. EGFR is a type of transmembrane glycoprotein receptor that functions as a tyrosine kinase (TK) (47). Its conformational changes can cause receptor polymerization and induce the activation of the intracellular TK subregion to activate multiple signaling pathways, including the PLC- $\gamma /$ PKCPI-3K/AKT, RAS-RAF-MEK-MAPK and STAT/ $\mathrm{NF}-\kappa \mathrm{B}$ A pathways $(47,48)$. In different cells or at various differentiation stages, the EGFR configuration changes to activate different signaling pathways, and the cells react to the activation or inhibition of a series of downstream molecules in the signaling pathway (49). This different signaling is in accordance with many other malignant tumors, such as gliomas and prostate cancer $(50,51)$. Moreover, EGFR mutations were found in cancerous and adjacent tissues of $10-40 \%$ of the lung cancer patients, among which $30 \%$ were Asian female non-smokers diagnosed with lung adenocarcinoma (52). miR-146a-5p was showed to be an important regulatory factor in tumor formation mediated by EGFR, which provided a new theoretical basis for the treatment of patients with lung cancer. Monoclonal antibody D2-40, a specific biomarker of lymphatic epithelial cells, can show via immunohistochemical staining the profiles of small lymphatic vessels stretching from the alveolar space to the small blood vessels in the lung lobules. As a result, this technique can be applied to the identification of lymphatic vessel tumor emboli $(53,54)$.

In this study, we conducted a target mRNA prediction of miR-146a-5p using in silico methods. When combining the results of twelve prediction-based databases, we obtained theoretical target genes of miR-146a-5p. However, this artificial prediction could have limitations in this single computational algorithm, and this process still needs further experimental verification. Based on the predicted genes, KEGG pathways and GO enrichment analysis were conducted. Pathways involved in cancer and toll-like receptor signaling were the most two significant pathway groups of the target genes. In addition, the protein localization of the mRNAs was enriched in the cell membrane and functioned in cellular processes. For molecular function, the predicted genes were associated with binding. Then, we could predict that miR-146a-5p may participate in tumor-related intercellular protein or nucleic acid binding signaling behavior. We plan to conduct further experimental research on miR-146a-5p regarding the development of tumorigenesis. For the PPI network, network nodes represent proteins, and edges represent protein-protein associations. We obtained 50 hub genes to input into the PPI and found that JUN, EGFR and RAC1 were the most relevant protein names among the selected possible targets of miR-146a-5p.

miR-146a-5p was overexpressed in an NSCLC cell line and could inhibit the tumorigenesis and angiogenesis in a CAM xenograft tumor model. The in silico analysis revealed that its target genes are found in pathways related to cancer. miR-146a-5p is a potential tumor suppressor gene in NSCLC. The carcinogenic mechanism and its prognostic value in lung cancer needs further research.

\section{Acknowledgements}

The study was supported by the funds of the National Natural Science Foundation of China (NSFC81360327, NSFC81560469), the Natural Science Foundation of Guangxi, China (2015GXNSFCA139009), Innovation Project of Guangxi Graduate Education (YCSZ2015106) and the Guangxi Zhuang Autonomous Region University Student Innovative Plan (No. 201610598003). The funders had no role in the study design, the data collection and analysis, the decision to publish, or the preparation of the manuscript. Wen-Ting Huang and Wei-Luan Cen contributed equally as co-first authors, and Xiao-Hua Hu and Gang Chen contributed equally as co-corresponding authors of this study.

\section{References}

1. Global Burden of Disease Cancer Collaboration, Fitzmaurice C, Dicker D, Pain A, Hamavid H, Moradi-Lakeh M, MacIntyre MF, Allen C, Hansen G, Woodbrook R, et al: The global burden of cancer 2013. JAMA Oncol 1: 505-527, 2015.

2. Dietel M, Bubendorf L, Dingemans AM, Dooms C, Elmberger G, García RC, Kerr KM, Lim E, López-Ríos F, Thunnissen E, et al: Diagnostic procedures for non-small-cell lung cancer (NSCLC): Recommendations of the European Expert Group. Thorax 71: 177-184, 2016.

3. Travis WD, Brambilla E, Müller-Hermelink HK and Harris CC (eds): World Health Classification of Tumours. Pathology and Genetics of Tumours of the Lung, Pleura, Thymus and Heart. IARC Press/Oxford University Press (distributor), Lyon, 2004.

4. Ul Hussain M: Micro-RNAs (miRNAs): Genomic organisation, biogenesis and mode of action. Cell Tissue Res 349: 405-413, 2012.

5. Bartel DP: MicroRNAs: Genomics, biogenesis, mechanism, and function. Cell 116: 281-297, 2004.

6. Zhang B, Pan X, Cobb GP and Anderson TA: microRNAs as oncogenes and tumor suppressors. Dev Biol 302: 1-12, 2007.

7. Baehrecke EH: miRNAs: Micro managers of programmed cell death. Curr Biol 13: R473-R475, 2003.

8. Ambros V: The functions of animal microRNAs. Nature 431: 350-355, 2004.

9. Michael MZ, O' Connor SM, van Holst Pellekaan NG, Young GP and James RJ: Reduced accumulation of specific mircoRNAs in colorectal neoplasia. Mol Cancer Res 1: 882-891, 2003.

10. Croce CM: Causes and consequences of microRNA dysregulation in cancer. Nat Rev Genet 10: 704-714, 2009.

11. Weber JA, Baxter DH, Zhang S, Huang DY, Huang KH, Lee MJ, Galas DJ and Wang K: The microRNA spectrum in 12 body fluids. Clin Chem 56: 1733-1741, 2010.

12. Bishop JA, Benjamin H, Cholakh H, Chajut A, Clark DP and Westra WH: Accurate classification of non-small cell lung carcinoma using a novel microRNA-based approach. Clin Cancer Res 16: 610-619, 2010.

13. Yanaihara N, Caplen N, Bowman E, Seike M, Kumamoto K, Yi M, Stephens RM, Okamoto A, Yokota J, Tanaka T, et al: Unique microRNA molecular profiles in lung cancer diagnosis and prognosis. Cancer Cell 9: 189-198, 2006.

14. Yu SL, Chen HY, Chang GC, Chen CY, Chen HW, Singh S, Cheng CL, Yu CJ, Lee YC, Chen HS, et al: MicroRNA signature predicts survival and relapse in lung cancer. Cancer Cell 13: 48-57, 2008.

15. Raponi M, Dossey L, Jatkoe T, Wu X, Chen G, Fan H and Beer DG: MicroRNA classifiers for predicting prognosis of squamous cell lung cancer. Cancer Res 69: 5776-5783, 2009.

16. Patnaik SK, Kannisto E, Knudsen S and Yendamuri S: Evaluation of microRNA expression profiles that may predict recurrence of localized stage I non-small cell lung cancer after surgical resection. Cancer Res 70: 36-45, 2010.

17. Jazdzewski K, Boguslawska J, Jendrzejewski J, Liyanarachchi S, Pachucki J, Wardyn KA, Nauman A and de la Chapelle A: Thyroid hormone receptor beta (THRB) is a major target gene for microRNAs deregulated in papillary thyroid carcinoma (PTC). J Clin Endocrinol Metab 96: E546-E553, 2011. 
18. Sun M, Fang S, Li W, Li C, Wang L, Wang F and Wang Y: Associations of miR-146a and miR-146b expression and clinical characteristics in papillary thyroid carcinoma. Cancer Biomark 15: 33-40, 2015.

19. Zheng T, Chou J, Zhang F, Liu Y, Ni H, Li X, Zheng L, Tang T, Jin L and Xi T: CXCR4 3'UTR functions as a ceRNA in promoting metastasis, proliferation and survival of MCF-7 cells by regulating miR-146a activity. Eur J Cell Biol 94: 458-469, 2015.

20. Xu B, Wang N, Wang X, Tong N, Shao N, Tao J, Li P, Niu X, Feng N, Zhang L, et al: miR-146a suppresses tumor growth and progression by targeting EGFR pathway and in a p-ERK-dependent manner in castration-resistant prostate cancer. Prostate 72 1171-1178, 2012.

21. Sun Q, Zhao X, Liu X, Wang Y, Huang J, Jiang B, Chen Q and $\mathrm{Yu} \mathrm{J}$ : miR-146a functions as a tumor suppressor in prostate cancer by targeting Rac1. Prostate 74: 1613-1621, 2014.

22. Xu B, Huang Y, Niu X, Tao T, Jiang L, Tong N, Chen S, Liu N, Zhu W and Chen M: hsa-miR-146a-5p modulates androgen-independent prostate cancer cells apoptosis by targeting ROCK1. Prostate 75: 1896-1903, 2015.

23. Li Y, Vandenboom TG II, Wang Z, Kong D, Ali S, Philip PA and Sarkar FH: miR-146a suppresses invasion of pancreatic cancer cells. Cancer Res 70: 1486-1495, 2010.

24. Yao Q, Cao Z, Tu C, Zhao Y, Liu H and Zhang S: MicroRNA-146a acts as a metastasis suppressor in gastric cancer by targeting WASF2. Cancer Lett 335: 219-224, 2013.

25. Kogo R, Mimori K, Tanaka F, Komune S and Mori M: Clinical significance of miR-146a in gastric cancer cases. Clin Cancer Res 17: 4277-484, 2011.

26. Hou Z, Yin H, Chen C, Dai X, Li X, Liu B and Fang X: microRNA-146a targets the L1 cell adhesion molecule and suppresses the metastatic potential of gastric cancer. Mol Med Rep 6: 501-506, 2012.

27. Sha M, Ye J, Zhang LX, Luan ZY and Chen YB: Celastrol induces apoptosis of gastric cancer cells by miR-146a inhibition of NF- $\kappa$ B activity. Cancer Cell Int 13: 50, 2013.

28. Zhou L, Zhao X, Han Y, Lu Y, Shang Y, Liu C, Li T, Jin Z, Fan D and $\mathrm{Wu} \mathrm{K}$ : Regulation of UHRF1 by miR-146a/b modulates gastric cancer invasion and metastasis. FASEB J 27: 4929-4939, 2013.

29. Wang RJ, Zheng YH, Wang P and Zhang JZ: Serum miR-125a-5p, miR-145 and miR-146a as diagnostic biomarkers in non-small cell lung cancer. Int J Clin Exp Pathol 8: 765-771, 2015.

30. Chen G, Umelo IA, Lv S, Teugels E, Fostier K, Kronenberger P, Dewaele A, Sadones J, Geers C and De Grève J: miR-146a inhibits cell growth, cell migration and induces apoptosis in non-small cell lung cancer cells. PLoS One 8: e60317, 2013

31. Li YL, Wang J, Zhang CY, Shen YQ, Wang HM, Ding L, Gu YC, Lou JT, Zhao XT, Ma ZL and Jin YX: MiR-146a-5p inhibits cell proliferation and cell cycle progression in NSCLC cell lines by targeting CCND1 and CCND2. Oncotarget 7: 59287-59298, 2016.

32. Mira E, Lacalle RA, Gómez-Moutón C, Leonardo E and Mañes S: Quantitative determination of tumor cell intravasation in a real-time polymerase chain reaction-based assay. Clin Exp Metastasis 19: 313-318, 2002

33. Busch C, Krochmann J and Drews U: The chick embryo as an experimental system for melanoma cell invasion. PLoS One 8: e53970, 2013.

34. Kunzi-Rapp K, Genze F, Küfer R, Reich E, Hautmann RE and Gschwend JE: Chorioallantoic membrane assay: Vascularized 3-dimensional cell culture system for human prostate cancer cells as an animal substitute model. J Urol 166: 1502-1507, 2001

35. Liu M, Scanlon CS, Banerjee R, Russo N, Inglehart RC, Willis AL, Weiss SJ and D'Silva NJ: The histone methyltransferase EZH2 mediates tumor progression on the chick chorioallantoic membrane assay, a novel model of head and neck squamous cell carcinoma. Transl Oncol 6: 273-281, 2013.
36. Ribatti D, Vacca A, Roncali L and Dammacco F: The chick embryo chorioallantoic membrane as a model for in vivo research on angiogenesis. Int J Dev Biol 40: 1189-1197, 1996.

37. Lokman NA, Elder AS, Ricciardelli C and Oehler MK: Chick chorioallantoic membrane (CAM) assay as an in vivo model to study the effect of newly identified molecules on ovarian cancer invasion and metastasis. Int J Mol Sci 13: 9959-9970, 2012.

38. Lester RD, Jo M, Montel V, Takimoto S and Gonias SL: uPAR induces epithelial-mesenchymal transition in hypoxic breast cancer cells. J Cell Biol 178: 425-436, 2007.

39. Chen PY and Long QC: Effects of cyclooxygenase 2 inhibitors on biological traits of nasopharyngeal carcinoma cells. Acta Pharmacol Sin 25: 943-949, 2004.

40. Huang da W, Sherman BT and Lempicki RA: Systematic and integrative analysis of large gene lists using DAVID bioinformatics resources. Nat Protoc 4: 44-57, 2009.

41. McGuire S: World Cancer Report 2014. Geneva, Switzerland: World Health Organization, International Agency for Research on Cancer, WHO Press, 2015. Adv Nutr 7: 418-419, 2016. doi: $10.3945 /$ an. 116.012211

42. Beasley MB, Dembitzer FR and Flores RM: Surgical pathology of early stage non-small cell lung carcinoma. Ann Transl Med 4: 238, 2016.

43. Di Leva G and Croce CM: Roles of small RNAs in tumor formation. Trends Mol Med 16: 257-267, 2010.

44. Weidhaas JB, Babar I, Nallur SM, Trang P, Roush S, Boehm M, Gillespie E and Slack FJ: MicroRNAs as potential agents to alter resistance to cytotoxic anticancer therapy. Cancer Res 67: 11111-11116, 2007.

45. Webster RJ, Giles KM, Price KJ, Zhang PM, Mattick JS and Leedman PJ: Regulation of epidermal growth factor receptor signaling in human cancer cells by microR NA-7. J Biol Chem 284: 5731-5741, 2009.

46. Yamaguchi G, Takanashi M, Tanaka M, Fujita K, Ohira T, Kuroda $M$ and Ikeda N: Isolation of miRNAs that target EGFR mRNA in human lung cancer. Biochem Biophys Res Commun 420: 411-426, 2012.

47. Jost M, Kari C and Rodeck U: The EGF receptor - an essential regulator of multiple epidermal functions. Eur J Dermatol 10: 505-510, 2000.

48. Ullrich A, Coussens L, Hayflick JS, Dull TJ, Gray A, Tam AW, Lee J, Yarden Y, Libermann TA, Schlessinger J, et al: Human epidermal growth factor receptor cDNA sequence and aberrant expression of the amplified gene in A431 epidermoid carcinoma cells. Nature 309: 418-425, 1984

49. Quadros MR, Peruzzi F, Kari C and Rodeck U: Complex regulation of signal transducers and activators of transcription 3 activation in normal and malignant keratinocytes. Cancer Res 64: 3934-3939, 2004

50. Rajan P, Elliott DJ, Robson CN and Leung HY: Alternative splicing and biological heterogeneity in prostate cancer. Nat Rev Urol 6: 454-460, 2009.

51. Nicholas MK, Lukas RV, Jafri NF, Faoro L and Salgia R: Epidermal growth factor receptor - mediated signal transduction in the development and therapy of gliomas. Clin Cancer Res 12: 7261-7270, 2006.

52. Shigematsu H and Gazdar AF: Somatic mutations of epidermal growth factor receptor signaling pathway in lung cancers. Int J Cancer 118: 257-262, 2006.

53. Van den Eynden GG, Van der Auwera I, Van Laere SJ, Colpaert CG, van Dam P, Dirix LY, Vermeulen PB and Van Marck EA: Distinguishing blood and lymph vessel invasion in breast cancer: A prospective immunohistochemical study. Br J Cancer 94: 1643-1649, 2006.

54. Kambouchner $\mathrm{M}$ and Bernaudin JF: Intralobular pulmonary lymphatic distribution in normal human lung using D2-40 antipodoplanin immunostaining. J Histochem Cytochem 57: 643-648, 2009 